\title{
Phase-Locked Arrays of Antiguides: Analytical Theory II
}

\author{
Dan Botez, Fellow, IEEE, Anatolii P. Napartovich, and Charles A. Zmudzinski, Member, IEEE
}

\begin{abstract}
By employing a variational technique on the eigenvalue equation for finite arrays of antiguides we obtain accurate analytical expressions for key parameters characterizing the adjacent array modes: the edge radiation loss, the loss caused by interelement losses, and the effective index. The upper adjacent mode at its maximum-loss point is found to be well approximated by the sum of two Bloch waves of wavenumbers $\pm \pi /[(X-$ 1) $\Lambda$ ], where $N$ is the element number, and $A$ is the array period. The intermodal discrimination, $\Delta \alpha$, between the adjacent mode and the resonant mode (at the adjacent-mode maximumloss point) is found to be well approximated $(<10 \%$ error) by $\alpha_{R R}$, the resonant-mode loss at resonance. Accurate analytical expressions are also derived for the two-dimensional opticalmode confinement factor $\Gamma$, and the dispersion between the resonant and adjacent modes. The obtained analytical formulas are discussed in light of device design, and general design rules are presented.
\end{abstract}

\section{INTRODUCTION}

$\mathbf{P}$ HASE-LOCKED arrays of antiguides [1]-[8] are a class of high-power coherent sources that have been quite successful in generating diffraction-limited (D.L.) powers well in excess of those available reliably from single-element, facet-passivated diode lasers (i.e., 150-200 mW). Using the phenomenon of resonant leaky-wave coupling [2], [3] recordhigh powers have been achieved from 20-element devices: (0.5-1.0) W CW D.L. beam operation, and $2 \mathrm{~W}$ pulsed D.L. beam operation [7]. Reliable operation at $0.5 \mathrm{~W} \mathrm{CW}$ output power has been obtained, without facet-mirror passivation, for over $3500 \mathrm{~h}$ [9]. Near-resonant arrays have provided in pulsed operation $5 \mathrm{~W}$ in beams with lobewidths $3 \times$ D.L. $(80 \mu \mathrm{m}$ wide aperture) [7], and $32 \mathrm{~W}$ in beams with lobewidths $2.5 \times$ D.L. (180 $\mu$ m-wide aperture) [8]. Furthermore, it has recently been shown both theoretically and experimentally [10] that quiescent (i.e., temporally stable) behavior can be obtained to powers as high as $0.45 \mathrm{~W} \mathrm{CW}$. By contrast to recently developed "broad-area"-type coherent devices: (flared) masteroscillator power amplifiers and unstable resonators, phaselocked antiguided arrays, due to their inherent strong built-in index profile, are neither affected by thermal- and carrierinduced refractive-index variations nor prone to filamentation.

Theory and experiment [1]-[5], [10], [11] have established that resonant leaky-wave coupling between antiguides is

Manuscript received August 15, 1994; revised September 29, 1994.

D. Botez is with the Department of Electrical Engineering, University of Wisconsin-Madison, Madison, WI 53706-1691 USA.

A. P. Napartovich is with Troitsk Institute for Innovation and Fusion Research, 142092 Troitsk, Moscow region, Russia.

C. A. Zmudzinski is with TRW Inc,, Redondo Beach, CA 90278 USA.

IEEE Log Number 9407796. much more effective for achieving stable in-phase lasing than evanescent-wave coupling between guides. A comprehensive theory for modal content and array-mode discrimination in antiguided arrays was developed [4], but it was solely based on numerical calculations. For device-design purposes it is of interest to have explicit formulas for array-mode eigenvalues and structure. By employing the technique of the translation matrix [12], we have already analyzed [13] resonant optical waveguide (ROW) arrays, and derived accurate analytical expressions for key parameters characterizing the resonant array modes. Here we derive, for the first time, accurate analytical expressions for key characteristics of array modes adjacent to the resonant modes (so called adjacent array modes): the radiation loss curve as a function of index step; the position of the radiation-loss maximum, the influence of interelement loss on these modes; the two-dimensional optical-mode confinement factor; and the effective index. As a result, one can determine analytically the intermodal discrimination between adjacent modes and resonant modes, and thus formulas are available for all parameters necessary for device design and optimization.

\section{ADJACENT-MODE LOSSES}

The effective-index profile under consideration is shown at the bottom of Fig. 1. For antiguides the active element has the real part of the index lower than that for the interelement region. The modulation of the imaginary part of index can be connected with the presence of interelement losses $\left(\alpha_{T}\right.$ in Fig. 1) or with the variation of the mode (transverse-field) overlap $\Gamma_{0}$ (top of Fig. 1). High gain in the low-index regions (i.e., high $\Gamma_{0}$ in the antiguide-core regions and/or $\alpha_{T}>0$ ) is what favors oscillation of the leaky array modes over the evanescent-wave array modes [7]. The translation matrix $T$ couples the amplitudes $a_{n}^{(1)}, a_{n}^{(2)}$ of functions corresponding to the scattering of lateral plane waves in the core of an antiguide [13]. For vectors consisting of these amplitudes the following equation can be obtained:

$$
a_{n+1}=T \bullet a_{n} .
$$

For an array of $N$ identical elements this equation can be solved:

$$
\boldsymbol{a}_{n}=\boldsymbol{T}^{n} \bullet \boldsymbol{a}_{0}
$$

Usually the radiation incident on a lateral boundary of the array is not reflected. In this case the boundary conditions have the 
form: $a_{0}^{(1)}=0$, and $a_{N}^{(2)}=0$. To employ these conditions we use the well known expression for $\boldsymbol{T}^{n}[13]$

$$
\boldsymbol{T}^{m}=\frac{\sin (n S)}{\sin S} \boldsymbol{T}-\frac{\sin (n-1) \cdot S}{\sin S} \boldsymbol{I},
$$

where $\cos S=\left(T_{11}+T_{22}\right) / 2$.

The lateral wave vectors, which correspond to the propagation in the antiguide core, $q$, and in the interelement region, $p$, are defined by the formulas:

$$
p=\left(k^{2} \bar{n}_{1}^{2}-\beta_{z}^{2}\right)^{1 / 2} \text { and } q=\left(k^{2} \bar{n}_{0}^{2}-\beta_{z}^{2}\right)^{1 / 2},
$$

where $k=\omega / c ; \bar{n}_{0}$ and $\bar{n}_{1}$ are the values of the complex effective-index expressions in the antiguide core and interelement region, respectively, and $\beta_{z}$ is the propagation constant of the array mode. The imaginary part of the index for a typical ROW device is much less than the real part, and the realindex difference also is much less than the index. It follows from (4) that $p$ and $q$ are coupled with each other through the relationship:

$$
p^{2}-q^{2}=k^{2}\left(\bar{n}_{1}^{2}-\bar{n}_{0}^{2}\right)
$$

The propagation constant can be written by using an effective index: $\beta_{z}=k \bullet n_{\text {eff }}+i \beta_{1}$, where $\left|\beta_{1}\right| \ll k \bullet n_{\text {eff }}$. Both $n_{\text {eff }}$ and $\beta_{1}$ are connected to the lateral wave vectors through the relationships:

$$
\begin{aligned}
n_{\mathrm{eff}} & =n_{0}-\left(1 / 2 n_{0}\right)\left(q_{R} / k\right)^{2}, \\
\beta_{1} & =-q_{R} \bullet q_{I} / k n_{0},
\end{aligned}
$$

where $q_{R}=\operatorname{Re} q, q_{I}=\operatorname{Im} q, n_{0}$ is the real part of $\bar{n}_{0}$, and $\Delta n=n_{1}-n_{0}$ is the lateral index step. From boundary conditions and the expression (3) the following dispersion equation can be obtained:

$$
T_{22}=\sin [(N-1) S] / \sin N S .
$$

After some algebra the following equivalents of (8) can be obtained:

$$
\begin{gathered}
T_{12} \bullet T_{21} \bullet \sin ^{2} N S / \sin ^{2} S=-1, \\
\cos N S=\left(T_{11}-T_{22}\right) \bullet i / 2\left(T_{12} \bullet T_{21}\right)^{1 / 2} .
\end{gathered}
$$

Any of the equations (8) to (10) can serve as a basis for further analysis.

Let us turn our attention to the case of the near-resonance condition. At the exact resonance the element region and the interelement spacing contain integer numbers of correspondent lateral half wavelengths. Denoting the lateral wavelength in the interelement spacing $\lambda_{1}$ (i.e., $\operatorname{Re} p \bullet \lambda_{1}=2 \pi$ ) the resonance condition is $s=m \lambda_{1} / 2 ; m=1,2, \cdots$. For the element region the resonance condition is $\operatorname{Re} q \bullet d=(1+l) \pi$, where $l=0$ corresponds to the fundamental element mode. For the near-resonance condition $|\sin q d|$ and $|\sin p s|$ are very small, and $|\cos q d|,|\cos p s|$ are close to unity. This allows the

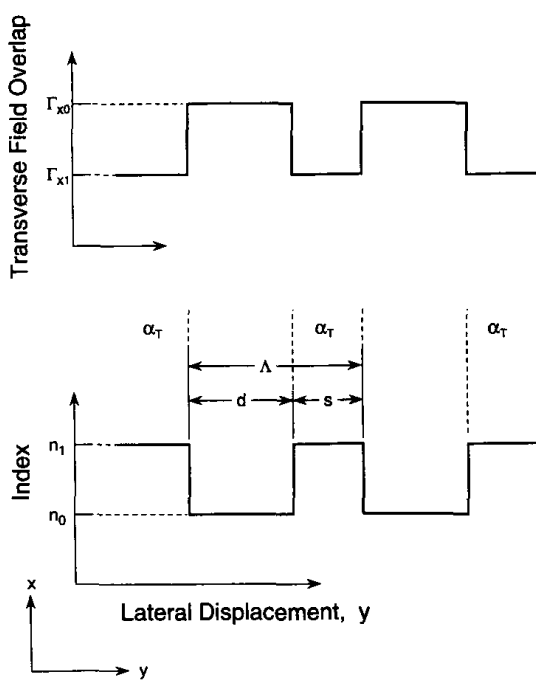

Fig. 1. Schematic representation of antiguided array. Top: lateral variation of the transverse optical-mode confinement factor. Bottom: lateral variation of the index of refraction. $\alpha_{T}$ is the interelement loss coefficient. $\Lambda$ is the array period.

simplification of (8) through (10), and to analyze the solutions explicitly.

Taking $p=p_{0}+p_{1} ; q_{0}+q_{1}$ ( $p_{0}$ and $q_{0}$ satisfy the resonance conditions, and $\left.\left|p_{1}\right| \ll p_{0} \cdot\left|q_{1}\right| \ll q_{0}\right)$ the expression for $S$ can be written down in the form [13]:

$$
\begin{aligned}
S & =(1+m+1) \pi+\left(q_{1}^{2} d^{2}+p_{1}^{2} s^{2}+2 \gamma p_{1} s \bullet q_{1} d\right)^{1 / 2} \\
& \equiv(1+m+1) \pi+F,
\end{aligned}
$$

$|F| \ll 1$, and (9) can be transformed to

$$
i \bullet x= \pm F / \sin N F
$$

where $x=q_{1} \bullet d \bullet\left(p_{0}^{2}-q_{0}^{2}\right) / 2 p_{0} q_{0}$. The problem of the correct selection of the sign in (12) will be discussed later. Using the relationship (5), a linear dependence of $p_{1}$ on $x$ can be found:

$$
p_{1} s=Y+2 \bullet x \bullet s /\left[d \bullet\left(p_{0}^{2} / q_{0}^{2}-1\right)\right]
$$

where $Y=k^{2} \bullet n_{0} \bullet \delta n \bullet s / p_{0}=\left(4 \pi \bullet n_{0} \bullet \delta n / m\right) \bullet(s / \lambda)^{2}$, where $\delta n$ is a complex quantity whose real part is an index differential, $\delta n_{R}=\Delta n-\Delta n_{0} .\left(\Delta n_{0}\right.$ is the lateral index differential at resonance.) Note that the $Y$ defined here is different from the $Y$ defined in [13], in that it does not contain $N$, the number of elements; and the $\delta n$ factor has both real and imaginary parts. Equations (11)-(13) for $F, x$ and $p_{1} \bullet s$ should be analyzed taking as a parameter the lateral index detuning from resonance. For the resonant mode this was realized in [13]. For the adjacent modes the conditions for an approximate theory to be applicable are more stringent. In what follows we assume strong optical-mode confinement for the antiguides. That means the condition $\left(p_{0}\right)^{2} \gg\left(q_{0}\right)^{2}$, which can be reformulated as

$$
(m \bullet d)^{2} \gg[s \bullet(1+1)]^{2} .
$$


It should be noted that the condition $d \gg s$ is highly desirable since it means good aperture filling, which in turn allows a large fraction of the power to reside in the central lobe of the far-field pattern [14]. Equation (11) can be transformed by substitution of (13) to a form more convenient for analysis:

$$
F^{2} \approx\left(Y+x \bullet \psi^{2} / 2\right) \bullet(Y+2 x),
$$

where $\psi=\left(2 q_{0} / p_{0}\right) \sqrt{1+(s / d)}$. At resonance $Y=0$, and from (12) and (14), one can obtain

$$
\sin N F= \pm i \psi
$$

wherefore it follows that for the resonant mode $\operatorname{Re} F_{0}=0$, Im $F_{0}=-i \psi / N$ in accordance with results of [13] (the sign in (15) can be chosen taking into account the connection between the quantity $F$ and the array-mode loss.) From (15) it also follows that there are adjacent modes having the eigenfrequency (i.e., the effective index) different from that for the resonant mode. $\operatorname{Re} F_{\text {adj }}= \pm \pi / N$, and $\operatorname{Im} F_{\text {adj }}=\operatorname{Im}$ $F_{0}=-i \psi / N$. (We restrict ourselves by considering only the nearest adjacent modes, because the condition of smallness of $|F|$ is broken at $N=10$ for the next modes.) It is a remarkable fact that at resonance the losses for the resonant mode and for the two adjacent modes are equal to each other. This result agrees with numerical calculations performed in [3] and [4].

$Y$ is proportional to the complex lateral-index detuning, the real part of which describes the deviation from the resonance, and the imaginary part is connected with the spatial modulation of loss and gain.

For strong optical-mode confinement $\psi \ll 1$, and $\left|\operatorname{Re} F_{\text {adj }}\right|$ $\gg\left|\operatorname{Im} F_{\text {adj }}\right|$. Let us consider (12) and (14) in the vicinity of the resonance point, where the real part of $F$ remains much greater than the imaginary part. In this region we approximate the sinfunction by a linear function: $\sin N F \approx(\pi-N F)$. Then the substitution of $F$ from (12) gives the algebraic equation

$$
\pi^{2} \xi^{2}=(\xi+i)^{2}(\xi+y / 2)\left(\psi^{2} \xi+2 y\right),
$$

where $\xi=N x, y=N Y$. It can be proved that $|\operatorname{Re} \xi| \gg|\operatorname{Im} \xi|$ in some vicinity of the resonance. Then one can solve (16) approximately. As a result we obtain:

$$
\operatorname{Re} \xi=(\pi / \psi)\left[\left(t^{2}+1\right)^{1 / 2}-t\right] \equiv(\pi / \psi) R
$$

where $t=(\operatorname{Re} y) / \pi \psi$, and

$$
\operatorname{Im} \xi=-2\left[1+N(\operatorname{Im} Y) R^{2} / \psi^{2}\right] /\left(1+R^{2}\right) .
$$

These solutions are applicable only when $|\operatorname{Re} \xi| \gg|\operatorname{Im} \xi|$. The adjacent-mode loss $\beta_{1}$ can be calculated (see (7)) from $\operatorname{Im} \xi$ by the formula

$$
\beta_{1_{\mathrm{adj}}}=-\left[(1+l)^{2} \lambda s /\left(m n_{0} N d^{3}\right)\right] \operatorname{Im} \xi \equiv-\beta_{0} \operatorname{Im} \xi
$$

where $\beta_{0}$ is the resonant-mode imaginary part of the dielectric constant at resonance. (Note that the $\beta_{0}$ defined here is $\beta_{0} / N$ of [13]).

Then:

$$
\beta_{1_{\text {adj }}} / \beta_{0}=2 \frac{1+N(\operatorname{Im} Y) R^{2} / \psi^{2}}{1+R^{2}}
$$

At resonance $t=0$ and $R=1$, and then for coupled fundamental (element) modes (i.e., $l=0$ ):

$$
\left(\beta_{1_{\text {adj }}} / \beta_{0}\right)_{\text {res }}=1+\frac{m^{2} d^{3}}{4 s^{2}(s+d)} N \operatorname{Im} Y
$$

By comparison for the resonant mode: $\left(\beta_{1} / \beta_{0}\right)_{\text {res }}=1+$ $(N / 3) \operatorname{Im} Y$. (see [13]). It follows that modulation of gain or loss has a much stronger influence (at and near resonance) on the adjacent-mode losses than on the resonant-mode losses. This fact and its implications will be discussed in the subsection on interelement loss.

The quantity $R$ depends on detuning from resonance for "upper" and "lower" adjacent modes in opposite direction, growing to one side and diminishing to the other side. Accordingly, the mode losses are diminishing or growing depending on the mode type and the sign of the detuning from the resonance. As was demonstrated numerically in [4], the adjacent-mode loss is maximum for a certain value of $\Delta n$. Such a behavior is not given by (20). Instead, as the detuning increases, the ratio of adjacent-mode loss to resonant-mode loss monotonically grows reaching an upper limit of 2 as $\delta n \rightarrow \infty$. This, of course, is not what happens when numerical calculations are performed [4]. However, the general trend is correct: away from resonance the adjacent-mode loss increases with increasing $\delta n$, and reaches a maximum which is almost twice the value of the resonant-mode loss at resonance [4]. To obtain an approximate value for the maximum adjacentmode loss one has to calculate (20) at the $\delta n$ value where the adjacent-mode loss reaches its maximum on numerically calculated plots [4].

\section{The MAXIMUM-Loss POINT}

The Bloch wavenumber, $q_{B}$, for an infinite-extent array with $l=0$ is 0 for the in-phase mode and $\pm \pi / \Lambda$ for out-of-phase modes. ( $\Lambda$ is the array period as shown in Fig. 1.) For an adjacent-like mode (e.g., mode 19 in [7]) the phase changes from one element to the other by $\pi /[(N-1) \Lambda]$, thus giving a $\pi / \Lambda$ total phase difference between the end elements. One could then assume that $q_{B, \text { adj }}=(\pi /(N-1) \Lambda)$. Furthermore, as it wil be shown below, it is safe to assume that the value of the adjacent-mode effective index at its maximum-loss point is equal to the effective index of the resonant mode at resonance. Then by using the eigenvalue equation from [13]:

$$
\cos \left(q_{B} \Lambda\right)=\cos q d \bullet \cos p s-\frac{p^{2}+q^{2}}{2 p q} \sin p s \bullet \sin q d
$$

for the adjacent mode, and taking into account that at resonance $p \bullet s=m \pi$ and $q \bullet d=(l+1) \pi,(22)$ becomes:

$$
\cos \left(\frac{\pi}{N-1}\right)=\cos \left(\frac{m \pi}{s} \Delta s_{m}\right)
$$

where $\Delta s_{m}$ is the deviation in $s$ with respect to $s_{\text {res }}=m \lambda_{1} / 2$, the resonance points. Then one obtains:

$$
\Delta s_{m}= \pm \frac{s_{\text {res }}}{m(N-1)} .
$$




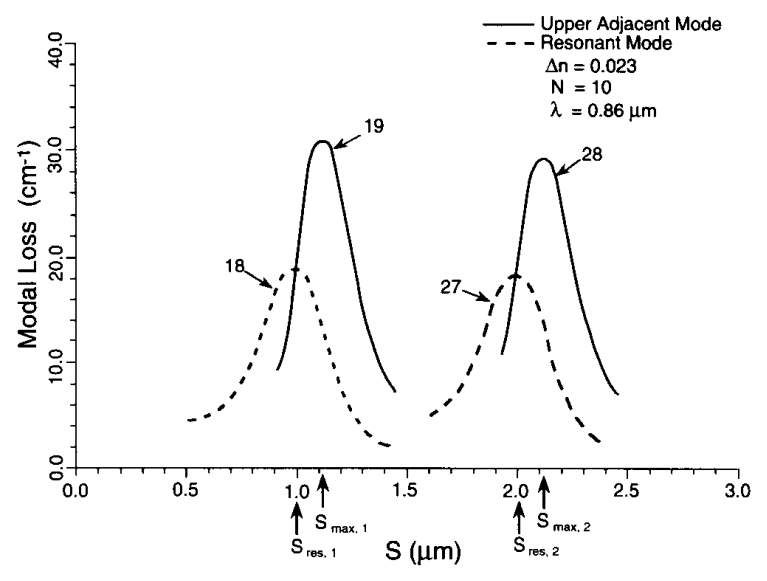

Fig. 2. Edge radiation losses for resonant and upper-adjacent modes of a 10-element array $(\Delta n=0.023 ; \lambda=0.86 \mu \mathrm{m})$ as a function of interelement spacing, $s . s_{\text {res }}$ are $s$ values corresponding to the resonances for each resonant mode, while $s_{\max }$ are $s$ values corresponding to the maximum-loss points for each upper-adjacent mode (see (25))

That is, for upper adjacent modes, the interelement spacing at their respective maximum-loss points is:

$$
\begin{aligned}
s_{\text {max }, m} & =s_{\text {res }}+\Delta s_{m}=s_{\text {res }}\left(1+\frac{1}{m(N-1)}\right) \\
& =\left(m+\frac{1}{(N-1)}\right) \frac{\lambda_{1}}{2} .
\end{aligned}
$$

Equation (25) is indeed a good approximation when we compare to numerical data for a 10-element array (Fig. 2). All (upper) adjacent-mode maxima occur a ninth of the way between subsequent resonance points. The same holds true for lower adjacent modes, but on the low- $s$ side of resonance.

However, if one employs the $q_{B \text {,adj }}$ and $s_{\max }$ values in the near-field amplitude expressions for array modes [15], the result is a curve without nulls.

We then realized that in effect the upper adjacent mode can be thought of being composed of the sum of two waves of Bloch wavenumbers $q_{B}=\pi /[(N-1) \Lambda]$ and $q_{B}=$ $-\pi /[(N-1) \Lambda]$, respectively. One sums the amplitudes of these two waves, using expressions from [15], and then squares to find the intensity. Shown in Fig. 3 is the result for the case: $N=11, d=3 \mu \mathrm{m}, s_{\max }=1.1 \mu \mathrm{m}, \Delta n=0.024$. Note that the intensity-profile envelope has a periodicity of $(N-1) \Lambda$. Over one period the mode looks virtually identical to the adjacent mode of a finite array at its maximum-loss point (upper right-hand and lower left-hand corners of Fig. 13 in [4]). This result validates our assumption that $n_{\text {eff }}$ for the adjacent mode at its maximum-loss point is virtually the same to the $n_{\text {eff }}$ of the resonant mode at resonance. Furthermore, the maximum-loss point for the upper adjacent mode(s) acquires a new meaning: it corresponds to the mode of an infiniteextent array composed of the sum of two waves of Bloch wavenumbers $q_{B}=\pi /[(N-1) \Lambda]$ and $-\pi /[(N-1) \Lambda]$, and of the same effective index as the single-antiguide index in the limit $\Delta n \rightarrow \infty$.

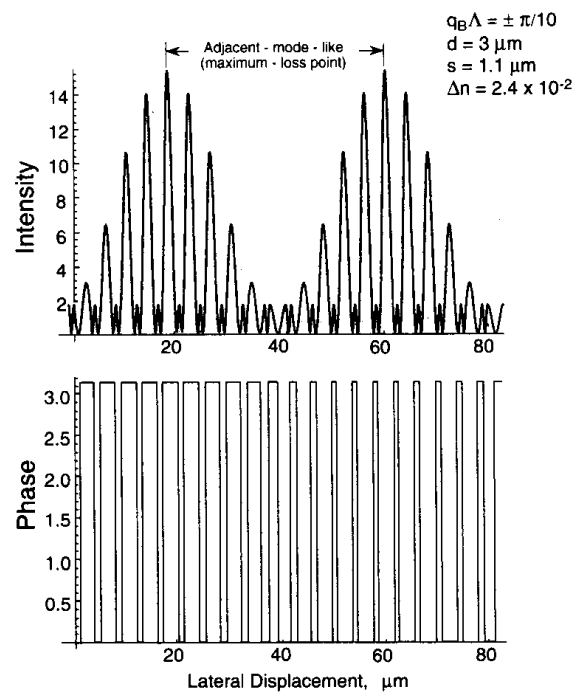

Fig. 3. Near-field intensity and phase profiles for a mode of an infinite-extent array. The mode is composed of the sum of two wavefunctions [15] of Bloch wavenumbers $\pi / 10 \Lambda$ and $-\pi / 10 \Lambda$, respectively. The field between two intensity-profile-envelope peaks is virtually identical to the field of the upper adjacent mode of an 11-element array at its maximum-loss point (for example, see Fig. 13 for $N=10$ in [4]).

Strictly speaking there is no specific reason why the adjacent modes' loss maxima should occur when their near-field profile is identical to the sum of Bloch wavefunctions. Having maximum field intensity at the array edges means maximum edge radiation losses only for devices with $N \geq 10$. A better definition is that the adjacent modes correspond to the sum of Bloch wavefunctions of wavenuber $\pm \pi /[(N-1) \Lambda]$ when the ratio of the peak field intensities in the end elements and the central interelement region(s) is $(m d / s)^{2}$ [17] (i.e., just as for resonant modes at resonance). For example, calculations on 3-element arrays [28] show that (25) does not correspond to adjacent-mode loss maxima, but rather to when $(\mathrm{md} / \mathrm{s})^{2}$ is the ratio of the peak field intensity in the end elements and the interelement regions.

Now that we know, for $N \geq 10$, where the maximum-loss points occur on a loss versus $s$ diagram (i.e., (25)) one can find the maximum-loss points on a loss versus $\Delta n$ diagram by using the relationship [4]:

$$
\Delta n_{\text {res }}=\frac{\lambda^{2}}{8 n_{0}}\left[\frac{m^{2}}{s^{2}}-\frac{(l+1)^{2}}{d^{2}}\right] .
$$

Upon differentiating (26), and taking into account that $N \gg 1$ one obtains:

$$
\delta \Delta n_{\text {res }}=\frac{-\lambda^{2}}{8 n_{0}} \frac{m^{2}}{s^{2}}\left(\frac{2 \Delta s}{s}\right) \cong \frac{-\lambda^{2}}{8 n_{0}} \frac{m^{2}}{s^{2}} \cdot \frac{2}{m N} .
$$

In the limit of strong confinement, we get

$$
\delta \Delta n_{\mathrm{res}}=\frac{-2 \Delta n_{\mathrm{res}}}{m N} .
$$

The negative shift in $\Delta n_{\text {res }}$ is the same as a positive shift in $\Delta n$ corresponding to a positive shift in $s$. Therefore, the 
difference in $\Delta n$ between the adjacent-mode maximum loss and resonance is:

$$
\delta n_{\mathrm{adj}, \max } \cong \frac{2 \Delta n_{\mathrm{res}}}{m N} .
$$

We find this equation to be in very good agreement with numerical data from [3] and [4]. (A more exact treatment by using (24) and (26) gives: $\delta n_{\text {adj, } \max }=$ $\delta n_{\text {res }}\left[(2 m N-1) /(m(N-1) \pm 1)^{2}\right]$, where + and - signs correspond to upper and lower adjacent modes, respectively. For $N \geq 10$ this expression is in excellent agreement with results from [3] and [4].)

It is interesting to note that for $\delta n=\delta n_{\text {adj, } \max }, \operatorname{Re} Y \cong$ $\pi / N$, which simplifies $(20)$ to:

$$
\begin{aligned}
\left(\beta_{1_{\mathrm{adj}}} / \beta_{0}\right)_{\mathrm{adj}, \max }= & \frac{\psi^{2}}{1+\psi^{2}-\sqrt{1+\psi^{2}}} \\
& \times\left(1+N \operatorname{Im} Y \frac{2+\psi^{2}-2 \sqrt{1+\psi^{2}}}{\psi^{4}}\right) .
\end{aligned}
$$

If $d / s>3$, which is in the spirit of strong confinement, with an error of $<10 \%$, (20) becomes:

$$
\begin{aligned}
\left(\beta_{1_{\mathrm{adj}}} / \beta_{0}\right)_{\mathrm{adj}, \max } & \cong 2\left(1-\frac{\psi^{2}}{4}\right)\left(1+\frac{N \operatorname{Im} Y}{4}\right) \\
& =2\left(1-\frac{s^{2}(l+1)^{2}}{m^{2} d^{3}} \Lambda\right)\left(1+\frac{N \operatorname{Im} Y}{4}\right) .
\end{aligned}
$$

This equation shows two relevant trends: 1) in the absence of interelement loss (i.e., $\operatorname{Im} Y=0$ ) the ratio of maximum adjacent-mode loss to the maximum resonant-mode loss approaches the value of 2 the stronger the confinement is; 2) at its maximum-loss point the adjacent mode, just as the resonant mode, is hardly affected by interelement loss. The latter feature is understandable, in that, just as for resonant mode at its resonance, the adjacent mode at its maximum-loss point has negligible interelement field [4].

\section{EDGE RADIATION LOSS AND INTERMODAL DISCRIMINATION}

In the case of no interelement loss (i.e., Im $Y=0$ ), the loss of the adjacent mode is purely edge radiation loss. From (20), we get

$$
\beta_{1_{\mathrm{adj}}} / \beta_{0}=\frac{2}{1+R^{2}} .
$$

We plot it for the case $N=20, m=1, l=0, d=3 \mu \mathrm{m}$, $s=1 \mu \mathrm{m}, \lambda=0.86 \mu \mathrm{m}$ in Fig. 4 . The approximation is very good from resonance ( $\Delta n=0.0245$ ) to the maximum-loss point $(\Delta n=0.027)$. At the maximum-loss point the error is $+7.9 \%$; that is the ratio $\beta_{1_{\text {adj }}} / \beta_{0}$ is 1.78 by comparison to the numerically calculated value of 1.65 . Table I displays numerically calculated and approximated values of $\beta_{1_{\text {adj }}} / \beta_{0}$ at the maximum-loss point.

With the sole exception the 10-element array of $d=3 \mu \mathrm{m}$, $s=1 \mu \mathrm{m}, m=1$, the error is at most $7.9 \%$. Two trends are

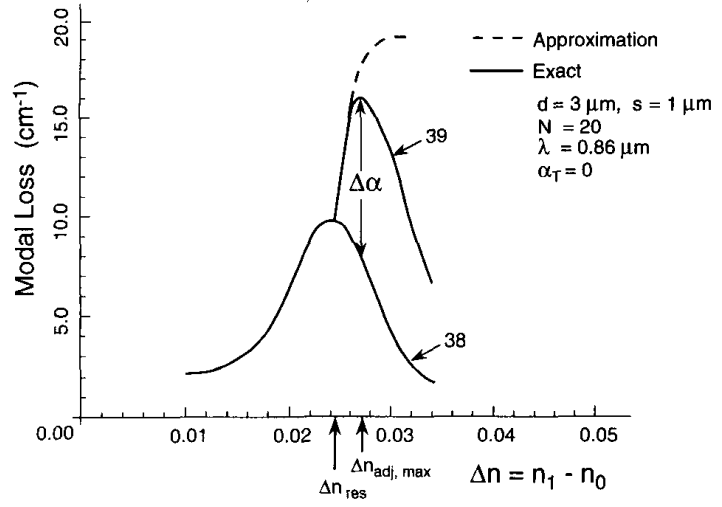

Fig. 4. Edge radiation loss versus lateral index step for a resonant mode $(L=38)$ and the upper adjacent mode $(L=39)$ of a 20-element array $(d=3 \mu \mathrm{m}, s=1 \mu \mathrm{m}, \lambda=0.86 \mu \mathrm{m})$ with no interelement loss. The dashed line corresponds to the approximation formula given by (32). At the adjacent-mode maximum-loss point (i.e., $\Delta n_{\text {adj.trlax }}$ ) the error is $7.9 \% . \Delta \alpha$ is defined as the intermodal discrimination.

TABLE I

Ratios of the Adjacent-Mode Maximum Radiation Loss to the Resonant-Mode Maximum Radiation Loss For Various ARray PARAmeters. THE APPRoXimated Values are ObTaINed From (20) with Im $Y=0$

\begin{tabular}{|c|c|c|c|c|c|c|}
\hline $\mathrm{N}$ & $\mathrm{d}(\mu \mathrm{m})$ & $\mathrm{s}(\mu \mathrm{m})$ & $\mathrm{m}$ & Exact & Approximation & Error \\
\hline 10 & 3 & 1 & 1 & 1.55 & $1.77^{*}$ & $14 \%$ \\
\hline 20 & 3 & 1 & 1 & 1.65 & $1.78^{*}$ & $7.9 \%$ \\
\hline 20 & 5 & 1 & 1 & 1.80 & 1.91 & $6.1 \%$ \\
\hline 10 & 3 & 1 & 2 & 1.80 & 1.93 & $7.2 \%$ \\
\hline 10 & 3 & 2 & 3 & 1.76 & 1.86 & $5.7 \%$ \\
\hline 10 & 3 & 2 & 4 & 1.80 & 1.91 & $6.1 \%$ \\
\hline 20 & 3 & 1.25 & 3 & 1.87 & 1.94 & $3.7 \%$ \\
\hline
\end{tabular}

${ }^{*}$ Calculated employing the exact value for $\delta n_{\text {adj.max }}$ (see comment after (29)). Basically in $(30) 4^{2}$ is multiplied by $(2 \mathrm{~N} /(2 N-1))^{2}$.

evident: better accuracy with higher number of elements, and with stronger confinement (i.e., higher $\mathrm{md} / \mathrm{s}$ ratio). Also, as predicted by (31), the ratio of losses is closer to 2 the larger the $m d / s$ ratio is (i.e., strong confinement).

The intermodal discrimination, $\Delta \alpha$, defined as the difference in loss between the adjacent-mode loss and the resonantmode loss at the point of maximum adjacent-mode loss (see Fig. 4), is given by

$$
\Delta \alpha=2\left(\beta_{1_{\text {adj,max }}}-\beta_{1_{\text {res }}}\left(\Delta n_{\text {adj,max }}\right)\right) .
$$

Its relative value with respect to the resonant-mode loss at resonance $\alpha_{R R}=2 \beta_{0}$ is

$$
\frac{\Delta \alpha}{\alpha_{R R}}=\frac{\beta_{1_{\text {adj,max }}}}{\beta_{0}}-\frac{\beta_{0}\left(\Delta n_{\mathrm{adj}, \max }\right)}{\beta_{0}} .
$$

By using (31) (i.e., strong confinement) with $\operatorname{Im} Y=0$, and the approximation formula for $\beta_{0}$ as a function of $\Delta n$ (i.e., $\beta_{0} / N$ as defined in [13]) one obtains for $N \geq 10$ :

$$
\frac{\Delta \alpha}{\alpha_{R R}} \cong 1.2-\frac{2 s^{2}(l+1)^{2}}{m^{2} d^{3}} \Lambda \text {. }
$$


Discounting the low confinement cases from Table I (i.e., the top two structures) one obtains for $\Delta \alpha / \alpha_{R R}$ values between 1.06 and 1.14. Taking into account that the error in calculating $\beta_{1_{\text {adj,max }}} / \beta_{0}$ varies between $+4 \%$ and $+8 \%$ it can be said that, with a maximum error of $10 \%$, one has:

$$
\Delta \alpha \cong \alpha_{R R}
$$

That is, the intermodal discrimination is to a good approximation equal with the resonant-mode loss at resonance. Since $\alpha_{R R} \cong \alpha_{R} / N$, where $\alpha_{R}$ is the single-antiguide loss, the derived relationship tells us very clearly the limitations of discriminating via edge radiation losses. The larger the number of elements and/or the larger $\Delta n$ value the smaller the effect of edge loss on intermodal discrimination will be. This explains why, at the present time, diffraction-limited beams are generally obtained for $N \leq 20$ and $\Delta n \leq 5 \times 10^{-2}$, at an emission wavelength $\lambda \cong 0.85 \mu \mathrm{m}$ [7]. Since $\Delta n_{\text {res }} \propto \lambda^{2}$ and $\alpha_{R R} \propto \lambda^{2} / \sqrt{\Delta n_{\text {res }}}$, it follows that $\Delta \alpha \propto \lambda$. That is, by increasing the emission wavelength the maximum number of elements for a diffraction-limited-beam array will increase accordingly. For instance, if $\lambda=1.55 \mu \mathrm{m}$ one can conceive of a 40-element array with $\Delta n=0.1, d=5 \mu \mathrm{m}$ and $s=1 \mu \mathrm{m}$ emitting in a diffraction-limited beam from a $240 \mu \mathrm{m}$-wide aperture.

Of course edge radiation loss is only one of the intermodal discrimination mechanisms in antiguided arrays [7]. In order to get diffraction-limited beams from arrays of $N>20$ and/or high $\Delta n$ values $(\Delta n \geq 0.1)$, one will have to resort to other discrimination mechanisms such as Talbot-type spatial filters [7] for near-resonant arrays or interelement loss and the $\Gamma$-effect [16] for perfectly resonant arrays.

\section{THE EFFECT OF INTERELEMENT LOSS}

Interelement loss is usually an effective way to suppress nonresonant out-of-phase modes [4]. However, close to resonance it helps suppress the adjacent modes as well [4], [18]. Now we can estimate this effect analytically.

Using (20) we plot in Fig. 5 the adjacent-mode losses for a case previously analyzed numerically (Fig. 17 in [4]): $N=10, d=3 \mu \mathrm{m}, s=1 \mu \mathrm{m}, \lambda=0.86 \mu \mathrm{m}$, and an interelement loss coefficient, $\alpha_{T}$, that varies between 70 and $75 \mathrm{~cm}^{-1}$ from resonance $(\Delta n=0.023)$ to the adjacent-mode maximum-loss point $(\Delta n=0.0276)$. For the (upper) adjacent mode 19 the approximation is good within $8 \%$ from resonance to the adjacent-mode maximum-loss point. At resonance there is large discrimination between the adjacent mode and the resonant mode. That is, as stated before, interelement loss affects the adjacent mode significantly more than the resonant mode. Taking $\operatorname{Im} Y=-\left(n_{0} s^{2} / m \lambda\right)(g-\alpha)$, where $g-\alpha$ is the net interelement gain, one obtains for the resonant mode (at resonance) the following expression for the ratio of modal loss with and without interelement loss:

$$
\left.\frac{\beta_{\text {res }}}{\beta_{0}}\right|_{\text {res }}=1+\frac{N}{3} \cdot \frac{n_{0} s^{2}}{m \lambda}(\alpha-g) .
$$

For the case shown in Fig. 5 one obtains a value of 1.093 . When plotting the computed $\beta$ res (dot in the figure) the value

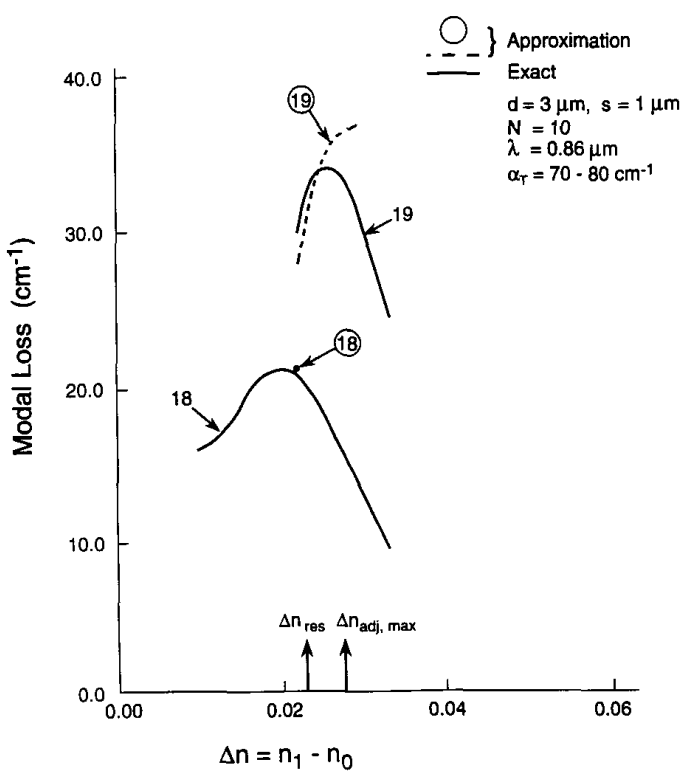

Fig. 5. Modal loss versus lateral index step for the resonant mode $(L=18)$ and the upper adjacent mode $(L=19)$ of a 10-element array $(d=3 \mu \mathrm{m}$, $s=1 \mu \mathrm{m}, \lambda=0.86 \mu \mathrm{m}$ ) with an interelement loss of $70-80 \mathrm{~cm}^{-1}$ (see Fig. 17 in [4]). The dashed line for mode 19 and the solid circle for mode 18 correspond to approximations given by (20) and by [13], respectively.

is found to be only $0.8 \%$ higher than the numerical value. For the adjacent mode at resonance, $(20)$, becomes

$$
\begin{aligned}
\left.\frac{\beta_{1_{\text {adj }}}}{\beta_{0}}\right|_{\text {res }} & =1+\frac{N}{\psi^{2}} \operatorname{Im} Y=1+\frac{N m^{2} d^{3}}{4 s^{2}(l+1)^{2} \Lambda} \operatorname{Im} Y \\
& =1+\frac{N m d^{3} n_{0}}{4(l+1)^{2} \lambda \Lambda}(\alpha-g) .
\end{aligned}
$$

For the case in Fig. 5 the value of $\beta_{1_{\mathrm{adj}}} / \beta_{0}$ is 1.467 . The calculated $\beta_{1_{\text {adj }}}$ value is $8 \%$ below the numerical value. In any event, the interelement loss has $\sim 5$ times stronger effect on the adjacent mode than on the resonant mode. That is because at and/or near resonance the adjacent mode has significant interelement field (see [4] and [18]).

It is instructive to look at the relative effect of interelement loss on adjacent and resonant modes by taking the ratio of the coefficients multiplying the $\operatorname{Im} Y$ quantity in the relative-loss equations (37) and (38). Then one obtains

$$
\left.\frac{\Delta \beta_{\text {adj }}}{\Delta \beta_{\text {res }}}\right|_{\text {res }}=\frac{3}{4} \frac{m^{2} d^{3}}{(l+1)^{2} s^{2} \Lambda},
$$

which is plotted in Fig. 6 as a function of element width for the case $m=1, l=0, s=1 \mu \mathrm{m}$. The curve is almost proportional with $(d / s)^{2}$. This behavior is a reflection of the fact that the amount of interelement field for the resonant mode decreases very fast as the $d / s$ ratio decreases [16]. In particular, for $d / s=5 / 1$, which are structures proven to give up to $75 \%$ of the energy in the central far-field lobe [14], the effect of interelement loss is 15 times stronger on the adjacent mode than on the resonant mode. This fact has 


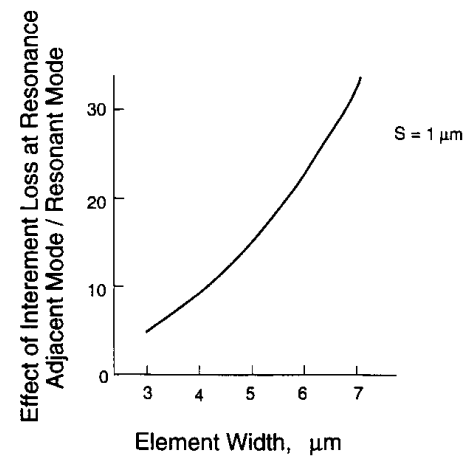

Fig. 6. The relative effect of interelement loss on the adjacent mode versus the resonant mode as a function of element width, $d$, for the case $m=1$, $l=0$ (see (39)). As $d$ increases the curve is well approximated by the expression $(3 / 4)(d / s)^{2}$.

allowed recently the design of a novel type of single-mode high-power device: the 3-core ARROW laser [19].

We also note from (39) the $m^{2}$ dependence of the relative influence of interelement loss. Structures with $m=3$ have been demonstrated [20] and proposed [21] as a means to obtain coherent arrays of relatively high index step $(\Delta n>0.1)$. It now appears that for such high- $\Delta n$ structures interelement loss could play a crucial role for intermodal discrimination. Assuming a high- $\Delta n$ structure with $d=3 \mu \mathrm{m}, s=1.25 \mu \mathrm{m}$, and $m=3$, the interelement loss impact is 27 times stronger on the adjacent mode compared to the resonant mode! Of course this effect happens only near resonance, but it could be a highly effective discriminator in 3-5-element devices, such as the 3-core ARROW [19], for which the range in $\Delta n$ over which interelement loss is a factor (i.e., $\Delta n \cong 4 \Delta n_{\text {res }} / m N$ ) is relatively large.

\section{THE $\Gamma$ EFFECT}

Analytical expressions have been deduced for the twodimensional optical-mode confinement factor, $\Gamma$, of resonant modes [16], but not for adjacent modes. We start by using a similar $\Gamma$ formula as employed in [16]:

$$
\Gamma=\left(1-\Gamma_{i}\right) \Gamma_{x 0}+\Gamma_{i} \Gamma_{x 1}
$$

where $\Gamma_{x 0}$ and $\Gamma_{x 1}$ are transverse optical-mode confinement factors in the element and interelement regions, respectively; and $\Gamma_{i}$ is the percentage of the adjacent-mode intensity that resides in the interelement regions. If interelement loss is present its contribution to the adjacent-mode loss is simply the product of $\Gamma_{i}$ and the loss coefficient in the interelement regions, $\alpha-g$. We can equate this product with the contribution of the interelement loss to the adjacent-mode loss as obtained from (20). That is,

$$
\Gamma_{i}(\alpha-g)=\frac{2 \beta_{0}}{1+R^{2}} N(\operatorname{Im} Y) \frac{R^{2}}{\psi^{2}} .
$$

After expressing $\operatorname{Im} Y$ the equation simplifies to

$$
\Gamma_{i}=\frac{4 \alpha_{R}}{1+R^{2}} \frac{n_{0} s^{2}}{m \lambda} \cdot \frac{R^{2}}{\psi^{2}} .
$$

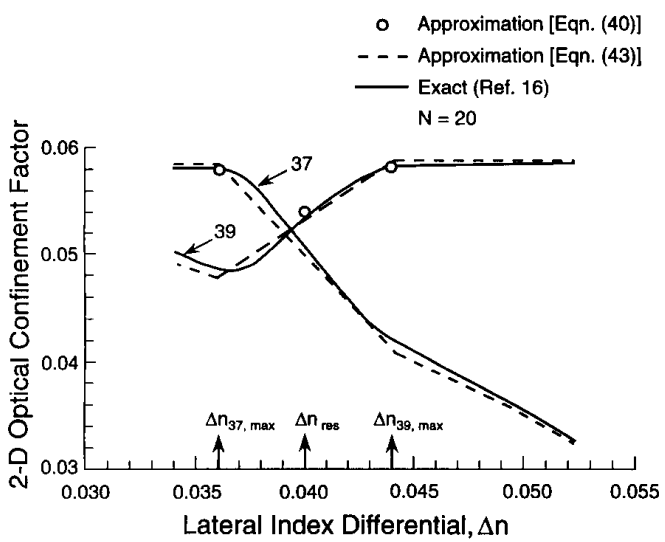

Fig. 7. The two-dimensional confinement factor for array modes adjacent to resonant mode $L=38$ in a 20-element array [16]. The open circles are approximations using (40)-(42). The dashed lines are approximations given by (43).

At the maximum-loss point for the adjacent mode $t=1 / \psi$, and thus (4lb) becomes

$$
\Gamma_{i}=\frac{4 \alpha_{R} n_{0} s^{2}}{m \lambda} \frac{2+\psi^{2}-2 \sqrt{1+\psi^{2}}}{\psi^{2}\left(1+\psi^{2}-\sqrt{1+\psi^{2}}\right)} .
$$

Using the case $N=20, d=3 \mu \mathrm{m}, s=1 \mu \mathrm{m}, \lambda=0.85 \mu \mathrm{m}$, $n_{0}=3.40 . \Delta n_{\text {res }}=0.04$ (i.e., [16]) one obtains a value of 0.08 for $\Gamma_{i}$. Reference [16] has $\Gamma_{x 0}=0.06$ and $\Gamma_{x 1}=0.03$, which using $(40)$, provides $\Gamma \approx 0.058$. This calculated value is plotted in Fig. 7 (open circles) and the agreement with the exact calculated values is excellent (i.e., $<1 \%$ ). At resonance we obtain $\Gamma=0.054$, also shown in Fig. 7 , which is only $1 \%$ higher than the numerical value.

Since the $\Gamma$ effect, just like the interelement loss, is a factor affecting adjacent modes only between resonance and their respective loss maxima, we propose the following approximation for $\Gamma$. For the upper adjacent mode:

$$
\begin{aligned}
& \Gamma=\Gamma_{10} \text { for } \Delta n \leq \Delta n_{\text {res }}\left(1-\frac{2}{m N}\right) \\
& \Gamma= \\
& \frac{\left(\Gamma_{01}-\Gamma_{10}\right) *\left(\Delta n-\Delta n_{\text {res }}\right)+\left(\Gamma_{01}+\Gamma_{10}\right)^{*}\left(2 \Delta n_{\text {res }} / m N\right)}{2 \Delta n_{\text {res }} / m N}
\end{aligned}
$$

for $\Delta n_{\text {res }}\left(1-\frac{2}{m N}\right)<\Delta n<\Delta n_{\text {res }}\left(1+\frac{2}{m N}\right)$

$\Gamma=\Gamma_{01}$ for $\Delta n \geq \Delta n_{\text {res }}\left(1+\frac{2}{m N}\right)$

where $\Gamma_{10}$ and $\Gamma_{01}$ are functions of $\Delta n$ for the infinite-extent array modes $(1,0)$ and $(0,1)$, respectively [16]. As shown in Fig. 7 this is an excellent approximation. Note that $\Gamma_{01}$ for $\Delta n \geq \Delta n_{\text {res }}(1+(2 / m N))$ can be replaced with a good deal of confidence by the $\Gamma$ value given by (40) and (42). Similar relationships hold for the lower adjacent mode, as evident from Fig. 7. 
The $\Gamma$ effect is a factor in intermodal discrimination only over a $4 \Delta n_{\text {res }} / m N$ range in $\Delta n$ around resonance. Then, just as for interelement loss, its usefulness as a discriminator against adjacent modes will be best exploited for arrays of low element number (3-5), such as the 3-core ARROW [19].

\section{THE EFFECTIVE INDEX}

Equation (6) gives a relationship for the effective index, $n_{\text {eff. }}$ Since $q_{R}=\operatorname{Re} q_{0}+\operatorname{Re} q_{1}, \operatorname{Re} q_{0}=\pi(l+1) / d$, and, $\operatorname{Re} q_{1} \ll \operatorname{Re} q_{0},(6)$ becomes

$$
n_{\text {eff }} \cong n_{0}-\frac{1}{2 n_{0}}\left(\frac{\lambda(l+1)}{2 d}\right)^{2}\left(1+2 \operatorname{Re} q_{1} \cdot d /(l+1) \pi\right)
$$

just as originally derived in [13]. Equation (17) gives us $\operatorname{Re} q_{1}$ :

$$
\operatorname{Re} q_{1}=\frac{\pi R}{\psi} \cdot \frac{2 p_{0} q_{0}}{p_{0}^{2}-q_{0}^{2}} \cdot \frac{1}{N d},
$$

which for the strong confinement condition (i.e., $p_{0}^{2} \geq q_{0}^{2}$ ) becomes

$$
\operatorname{Re} q_{1} \cong \frac{\pi R}{\psi} \cdot \frac{2(l+1) s}{m d} \cdot \frac{1}{N d}
$$

Inserting $\operatorname{Re} q_{1}$ in (44) gives

$$
n_{\text {eff }} \cong n_{0}-\frac{1}{2 n_{0}}\left(\frac{\lambda(l+1)}{2 d}\right)^{2}\left(1+\frac{4 s}{m d N} \cdot \frac{R}{\psi}\right) .
$$

This is the analytical formula for adjacent-mode effective index. We study and compare it with numerical calculations [22] at two points: maximum-loss and resonance.

At the maximum-loss point $t=1 / \psi$ and $R$ becomes $\left(\pi / \psi^{2}\right)\left(\sqrt{1+\psi^{2}}-1\right)$. For the strong-confinement condition $R=\pi / 2$. (Even in the extreme case $d / s=3, m=1, l=0$, the error introduced by making this approximation is only $13 \%$. All cases of practical interest give smaller errors.) Then (46) becomes

$$
\left.n_{\mathrm{eff}}\right|_{\mathrm{adj}, \max }=n_{0}-\frac{1}{2 n_{0}}\left(\frac{\lambda(l+1)}{2 d}\right)^{2}\left(1+\frac{2 s}{m d N}\right) .
$$

The quantity $n_{0}-\left(1 / 2 n_{0}\right)(\lambda(l+1) / 2 d)^{2}$ is simply the effective index of the resonant mode at resonance [13], which is also the $n_{\text {eff }}$ for a single antiguide in the limit $\Delta n \rightarrow \infty$ [4]. For a simple case such as $n_{0}=3.4, s=1 \mu \mathrm{m}, d=3 \mu \mathrm{m}$, $m=0, l=1$, and $N=10$, the deviation of $\left.n_{\mathrm{eff}}\right|_{\mathrm{adj}, \max }$ from $n_{\text {eff,res }}$, is only 0.0002 . That is, our assumption that $\left.n_{\text {eff }}\right|_{\text {adj,max }} \cong n_{\text {eff,res }}$ was well founded. In the limit $N \rightarrow \infty$ the two quantities are identical, as considered in Section III. Excellent agreement is found with numerical results [22] for the above-mentioned case (i.e., a 3.39980 calculated value versus a 3.39967 numerically obtained value).

At resonance $R=1$, and (46) becomes

$$
n_{\mathrm{eff}}=n_{0}-\frac{1}{2 n_{0}}\left(\frac{\lambda(l+1)}{2 d}\right)^{2}\left(1+\frac{2}{(l+1) N \sqrt{1+\frac{s}{d}}}\right) .
$$

This is also in excellent agreement with numerical data [22] (3.39946 calculated value versus 3.39944 numerically obtained value). We can now obtain a quantity of interest, the difference in effective index between the resonant mode and the adjacent mode at resonance:

$$
\left.\delta n_{\mathrm{eff}}\right|_{\mathrm{res}}=\frac{\lambda^{2}(l+1)}{4 n_{0} N d^{3 / 2} \sqrt{\Lambda}} .
$$

Equation (49) gives in effect the dispersion. For the case $l=0, N=10, s=1 \mu \mathrm{m}, d=3 \mu \mathrm{m}, l=0.86 \mu \mathrm{m}, n_{0}=3.4$, one gets $\delta n_{\text {eff }}=5.2 \times 10^{-4}$, only $4 \%$ less than the figure obtained from the numerical plots of [22]. In this case $\delta n_{\text {eff }}$ corresponds to a wavelength dispersion of $0.23 \AA$, a typical value for phase-locked arrays. As expected the dispersion is inversely proportional with the number of array elements.

Finally, the dispersion at the adjacent-mode loss maximum can be obtained by using the $n_{\text {eff }}$ formula for the resonant mode ((26) in [13]), (29), and (47):

$$
\left.\delta n_{\mathrm{eff}}\right|_{\mathrm{adj}, \max }=\frac{\lambda^{2}(l+1)^{2}}{4 n_{0} d^{2}} \cdot \frac{5 s}{3 m d N} .
$$

It is immediately evident this quantity (for the case $l=0$ ) is smaller by at least a factor of 1.5 than the $\delta n_{\text {eff }}$ value at resonance (i.e., (49)). That is, typical dispersion values for 10 element arrays with $\Delta n$ at the adjacent-mode loss maximum will be around $0.15 \AA$. There is experimental evidence that this value for dispersion is in the right ballpark for antiguided arrays. Specifically Van de Ziel et al. have recently obtained [23] a dispersion value of $0.15 \AA$ for GaAs 13-element gainguided arrays, which after all are antiguided arrays.

\section{CONCLUSION}

Considering our previous work [13], and the results obtained here we have obtained analytical approximations for all parameters affecting intermodal discrimination in (longitudinally uniform) antiguided arrays: the 2-D optical-mode confinement factor $\Gamma$, the edge radiation loss, and the interelement loss. It is now a simple exercise to compute the threshold-current densities for the various array modes [24], [25], and determine over what range in $\Delta n$ is the desired mode, the in-phase resonant mode, favored.

There is still the issue of how to compute losses for arrays with Talbot-type spatial filters [24]. As pointed out in [24] a good approximation for the resonant-mode transmissivity can be obtained from the work of Leger et al. [26]. For adjacent modes the situation is more complicated. One either has to use beam propagation through the filter and compute the overlap integral [4], [18], or approximate the adjacent-mode envelope as two adjacent half raised-cosine curves (see Fig. 3) and weigh this fact in Leger's formula [26]. In any event, Talbot-type filters are not absolutely necessary [6] if the proper mixture of intrinsic antiguided-array modal discrimination mechanisms is employed.

The analytic formulas derived here not only help in device design, but also provide some useful design guidelines. In order to obtain diffraction-limited-beam operation to high powers: 
1) For arrays of large element-number $(N=20-40)$, one has to employ either relative low $\Delta n$ values $(3-5 \times$ $10^{-2}$ ) or, if technologically feasible, operate right at resonance in devices with large interelement loss. Increasing the emission wavelength will proportionally increase the maximum number of elements for coherent operation.

2) For arrays of low element number $(N=3-5)$, high$\Delta n$ values $\left(\cong 10^{-1}\right)$ coupled with large interelement loss (sim200 $\mathrm{cm}^{-1}$ and large element width/interelement spacing ratios $(>3)$ are preferred, as long as ARROWtype terminations are provided.

The latter are particularly attractive since the resonance condition is significantly eased, and thus it should be relatively easy to achieve $1 \mathrm{~W}$ coherent power from $20 \mu \mathrm{m}$-wideaperture devices [19], [29]. Furthermore, 3-core ARROW devices can be used as efficient master oscillators for antiguided MOPA's [19], [27], [29], which hold the promise for 3-5 W coherent $\mathrm{CW}$ power without the inherent problems (filamentation; drive-dependent astigmatism) that plague broad-area (flared) MOPA's [25].

Note Added in Proof: We have recently realized that for arrays of small element number (i.e., $N<10$ ), the factor $N$ multiplying $\operatorname{Im} Y$ in formulas expressing the effect of interelement loss should be replaced by $N-1$. This fact has been confirmed by comparing results of analytical calculations to results of numerical calculations for 3-element arrays with interelement loss [28].

\section{REFERENCES}

[1] D. Botez, L. Mawst, P. Hayashida, G. Peterson, and T. J. Roth, "Highpower, diffraction-limited-beam operation from phase-locked diodelaser arrays of closely spaced 'leaky' waveguides (antiguides)," Appl Phys. Lett., vol. 53, pp. 464-466, Aug. 1988.

[2] D. Botez, L. J. Mawst, and G. Peterson, "Resonant leaky-wave coupling in linear arrays of antiguides," Electron. Lett., vol. 24, pp. 1328-1330, Aug. 1988.

[3] D. Botez, L. J. Mawst, G. Peterson, and T. J. Roth, "Resonant optical transmission and coupling in phase-locked diode laser arrays of antiguides: The resonant optical waveguide array," Appl. Phys. Lett., vol. 54 , pp. 2183-2185, May 1989.

[4] - "Phase-locked arrays of antiguides: Modal content and discrimination," IEEE J. Quantum Electron., vol. 26, pp. 482-495, Mar. 1990.

[5] G. R. Hadley, "Two-dimensional waveguide modeling of leaky-mode arrays," Opt. Lett., vol. 14, pp. 859-861, Oct. 1989.

[6] G. R. Hadley, D. Botez, and L. J. Mawst, "Modal discrimination in leaky-mode (antiguided) arrays," IEEE J. Quantum Electron., vol. 27, pp. 921-930, Apr. 1991.

[7] D. Botez, "High-power monolithic phase-locked arrays of antiguided semiconductor diode lasers," Proc. Inst. Elec. Eng. Part J, Optoelectronics, vol. 139, pp. 14-23, Feb. 1992.

[8] D. Mehuys, J. Major, Jr., and D. F. Welch, "High-power, high-efficiency antiguided laser arrays," in SPIE Proc. OE-LASE '93 Meeting, Los Angeles, CA, vol. 1850, pp. 2-12, Los Angeles, CA, 1993.

[9] L. J. Mawst, D. Botez, M. Jansen, T. J. Roth, C. Zmudzinski, C. Tu, and J. Yun, "Resonant-optical-waveguide antiguided diode laser arrays," in SPIE Proc. OE-LASE '92 Meeting, vol. 1634, 1992, pp. 2-12.

[10] S. Ramanujan, H. G. Winful, M. Felisky, R. K. DeFreez, D. Botez, M. Jansen, and P. Wisseman, "Temporal behavior of resonant-opticalwaveguide phase-locked diode laser arrays," Appl. Phys. Lett., vol. 64 pp. 827-829, Feb. 1994

[11] R. Li and T. Erneux, "Stability conditions for coupled lasers: Series coupling versus parallel coupling," Opt. Commun., vol. 99, pp. 196-200, 1 June 1993.
[12] P. Yeh, Optical Waves in Layered Media. New York: Wiley, 1988.

[13] D. Botez and A. P. Napartovich, "Phase-locked arrays of antiguides: Analytical theory," IEEE J. Quantum Electron., vol. 30, pp. 975-980, Apr. 1994.

[14] C. Zmudzinski, D. Botez, and L. J. Mawst, "Coherent, one-watt operation of large-aperture resonant arrays of antiguided diode lasers," Appl. Phys. Lett., vol. 62, pp. 2914-2916, June 1993.

[15] D. Botez and T. Holcomb, "Bloch-function analysis of resonant arrays of antiguided diode lasers," Appl. Phys. Lett., vol. 60, pp. 539-541, Feb. 1992.

[16] D. Botez and L. J. Mawst, " $\Gamma$ effect: Key intermodal-discrimination mechanism in arrays of antiguided diode lasers," Appl. Phys. Lett., vol. 60, pp. 3096-3098, 1992.

[17] For an adjacent mode with the field peaked at the array edges the phase has to change by $2 \pi N$ across an $N$-element array (for $m=1$ ) in order that the fields in the end elements be out of phase. The array size is $d N+(N-1)\left(s+\Delta s_{m}\right)=(d+s) N$. That is, each $2 \pi$ phase shift corresponds to a lateral distance of $d+s$. Starting from the left edge (in Fig. 3) it means that the fields in the first element and interelement regions correspond to half-sine curves of $d$ - and swide basewidth, respectively. Therefore, the ratio of their peak field intensities should be $(d / s)^{2}$, as confirmed in Fig. 3. Similarly, for an even-element-number array the field in the central interelement region is mostly a half-cosine curve of basewidth equal to $s$, as expected since 3 is the same as for the resonant mode. Again this means a $(d / s)^{2}$ ratio between the end-element(s) peak field intensity and the central interelement-region(s)' peak field intensity. (Actually, as evident from Fig. 3, the ratio of peak field intensity between end-element regions and all interelement regions is $(d / s)^{2}$.) For a finite-size array the field profile is composed of both a standing-wave part and a traveling-wave part (i.e., the edge radiation losses). The latter is negligible only near the array center $[4]$, for which reason the $(d / s)^{2}$ value holds only for the ratio of peak field intensities in the end elements and the central interelement region(s) (see upper right-hand corner and lower left-hand corner in Fig. 13 of [4]).

[18] P. D. Van Eijk, M. Reglat, G. Vasilieff, G. J. M. Krijnen, A. Driessen, and A. J. Mouthaan, "Analysis of modal behavior of an antiguide diode laser array with Talbot filter," J. Lightware Technol., vol. 9, pp. 629-634, 1991.

[19] C. Zmudzinski, D. Botez, L. J. Mawst, and R. F. Nabiev, "Three-core ARROW-type diode laser: Novel high-power, single-mode device and effective master oscillator for flared antiguided MOPAs," in Tech. Dig. CLEO-Europe Conf., Paper CTuP3, Amsterdam, The Netherlands, Aug. 28-Sept. 21994.

[20] J. S. Major, Jr. D. Mehuys, D. F. Welch, and D. Scifres, "High-power high efficiency antiguide laser arrays," Appl. Phys. Lett., vol. 59, pp. 2210-2212, Oct. 1991.

[21] R. F. Nabiev, X. Yi, P. Yeh, and D. Botez, "Self-stabilization of the fundamental in-phase mode in resonant antiguided lasers," in Proc. SPIE OE-LASE '93 Meeting, Los Angeles, CA, Jan. 16-23, 1993, vol. 1850 , pp. 23-36, 1993; also accepted in an extensively revised version for publication in the June 1995 issue of IEEE J. Select. Topics Quantum Electron.

[22] C. A. Zmudzinski, D. Botez, and L. J. Mawst, "Simple description of laterally resonant, distributed-feedback-like modes of arrays of antiguides," Appl. Phys. Lett., vol. 60, pp. 1049-1051, Mar. 1992.

[23] J. P. Van der Ziel, X. F. Tang, and A. K. Chin, "Observation of complete set of array modes of single-quantum-well gain-guided GaAs laser arrays," in Proc. OSA Annu. Meeting, Paper WTT3, Dallas, TX, Oct. 2-7 1994

[24] D. Botez, M. Jansen, L. J. Mawst, G. Peterson, and T. J. Roth, "Wattrange, coherent, uniphase powers from phase-locked arrays of antiguide diode lasers," Appl. Phys. Lett., vol. 58, pp. 2070-2072, May 1991.

[25] D. Botez and D. R. Scifres, Eds. Diode Laser Arrays. Cambridge, UK: Cambridge Univ. Press, 1994.

[26] J. R. Leger and M. P. Griswold, "Binary-optics miniature Talbot cavities for laser beam addition," Appl. Phys. Lett., vol. 56, pp. 4-6, Jan. 1990.

[27] D. Botez, M. Jansen, C. Zmudzinski, L. J. Mawst, P. Hayashida, C. Tu, and R. F. Nabiev, "Flat-phasefront fanout-type power amplifier employing resonant-optical-waveguide structures," Appl. Phys. Lett., vol. 63, pp. 3113-3115, Dec. 6, 1993.

[28] D. Botez and C. Zmudzinski, unpublished work.

[29] C. Zmudzinski, D. Botez, L. J. Mawst, A. Bhattacharya, M. Nesnidal, and R. F. Nabiev, "Three-core ARROW-type diode laser: Novel highpower, single-mode device, and effective master oscillator for flared antiguided MOPAs," J. Select. Topics Quantum Electron., vol. 1, no. 2 , June 1995 (to appear). 


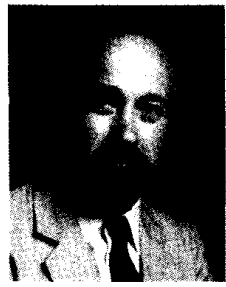

Dan Botez, (S'71-M'79-SM'85-F'86) was born in Bucharest, Romania, on May 22, 1948. He received the B.S. degree with "highest honors," and the M.S. and Ph.D. degrees in electrical engineering from the University of California, Berkeley, in 1971, 1972 and 1976, respectively. As part of his graduate work, he studied the characteristics of AlGaAs-GaAs layered structures grown by liquid-phase expitaxy over channeled substrates, as well as novel diode lasers made possible by this method.

For one year following graduation, he continued his research in the semiconductor-laser field as Postdoctoral Fellow at the IBM T. J. Watson Research Center, Yorktown Heights, NY. In 1977, he joined RCA Laboratories, Princeton, NJ, where his research concentrated on new types of high-power, single-mode AlGaAs-GaAs semiconductor lasers. Such devices became commercial products and were instrumental in the firs demonstration of high-data-rate optical recording with diode lasers (1981). From 1982 to 1984, he was Research Leader in the Optoelectronics Group. In 1983 , his research interests shifted to phase-locked diode-laser arrays, and he was part of the team that developed the coupled-mode formalism for evanescent-wave-coupled arrays. In 1984, he joined Lytel, Inc., as Director of Device Development, in which capacity he led the development of various types of optoelectronic devices for the fiber-optical communications. In 1986 he joined TRW, Inc., Redondo Beach, CA, where his responsibilities were Chief Scientist and Head of the Electro-Optics Research Center, and Senio Staff Scientist in TRW's Research Center. In 1990, he was chosen Technical Fellow of TRW. At TRW, his research resulted in the development of nove types of high-power, diffraction-limited phase-locked arrays. In 1990, he led the team that broke the $1-\mathrm{W}$ coherent-power barrier for semiconductor lasers. In 1992, he co-developed the Bloch-function analysis for antiguided arrays. In 1993, he becaome Professor and holder of the Philip Dunham Reed Chair in Electrical Engineering at the University of Wisconsin-Madison. He has over 160 technical publications, holds 31 patents, and co-authored two books

Dr. Botez is a member of Phi Beta Kappa, the Optical Society of America and of the IEEE/LEOS Board of Governors. In 1979, he received an RCA Outstanding Achievement Award for contributions to the development of high-density optical recording system employing a diode laser. As part of the events associated with the IEEE centennial year (1984), he was chosen the Outstanding Young Engineer of LEOS, and was awarded an IEEE Centennia Key to the future. In 1985, he was elected IEEE Fellow "for leadership in the development of high-power semiconductor lasers." In 1990, he received a TRW Chairman's Award for Innovation for his work on high-power coheren diode-laser arrays. In 1994, he was awarded the "Doctor Honoris Causa" honorary degree by the Polytechnical University of Bucharest, Romania.

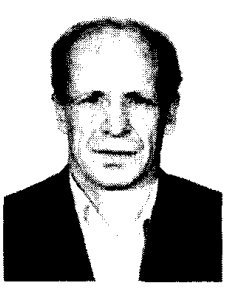

Anatolii P. Napartovich was born in Ulan-Ude, Russia, on December 1, 1940. He graduated from the Moscow Institute of Physics and Technology in 1964, and received Kandidat and Doctor of Science degrees in plasma physics from the HighTemperature Institute (Moscow) in 1969 and from Kurchatov Atomic Energy Institute in 1980, respectively.

His kandidat dissertation was devoted to the res onance radiation transfer theory. In 1967, he joined Kurchatov Atomic Energy Institute. After the kandidat dissertation defense his research concentrated mainly on the theory of a gas discharge and resonator optics for fast-flow $\mathrm{CO}_{2}$ lasers. From 1967 to 1972 and from 1972 to 1982 , he was junior researcher and senior researcher, respectively, in the Branch of Kurchatov Institute. He predicted several kinds of plasma and optical instabilities limiting the power and optical quality of laser beams. Under his guidance the comprehensive computer models for $\mathrm{CO}_{2}$ $\mathrm{CO}$, and excimer lasers were formulated and successfully used for theoretical description of these lasers. Since 1982, he is a head of the Gas Laser Theory Lab. Recently his interests shifted to the theory of optically coupled laser arrays. He proposed a new type of coupling based on the Talbot effect, which was realized in an experiment. He is involved also in the work on the glow discharge in an air flow at atmospheric pressure, which is very promising for applications in flue-gas cleanup. He has over 200 technical publications and holds six inventions. Since 1973, he delivered also lectures in laser physics for students of Moscow Institute of Physics and Technology.

Three times Dr. Napartovich received the Kurchatov Award and once the State Prize. He is a member of the Editorial Board of the Soviet Journal of Quantum Electronics and a memeber of SPIE Rus.

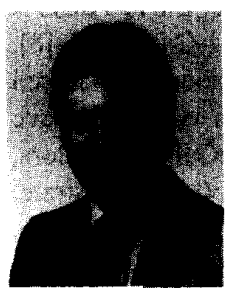

Charles A. Zmudzinski (S'84-M'89) was born in South Bend, IN, in 1962. He received the B.S. degree in electrical engineering from the University of Notre Dame in 1984. He received the M.S and Ph.D. degrees in electrical engineering from the University of Illionois in 1986 and 1989 respectively.

His research focused on the development of high-power diode lasers and laser arrays grown by MOCVD. In 1989, he was a postdoctoral research associate at the University of Florida, where he studied the temperature dependence of threshold current and differential quantum efficiency of quantum-well lasers, differential gain in quantum-well lasers, and II-VI semiconductor materials for diode lasers emitting in the bluegreen spectral region. In 1991, he joined TRW, where he has contributed to the development of phased-locked arrays of antiguided diode lasers for highpower coherent operation, and high-power single-stripe diode lasers based on the ARROW concept. At TRW, he is presently studying semiconductor optical amplifiers and modulators for high-speed analog applications. He has authored or co-authored more than 30 technical papers. 